\title{
GINÁSTICA LABORAL NA PREVENÇÃO DA LOMBALGIA (OCUPACIONAL) NO AMBIENTE DE TRABALHO: revisão
}

\section{integrativa}

DOI: $10.48140 /$ digitaleditora.2020.002.3

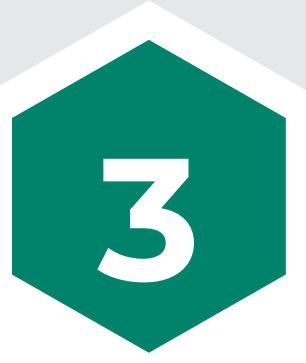

RESUMO

Objetivos: O objetivo deste estudo constitui em analisar através da revisão bibliográfica, a importância do programa da ginastica laboral na prevenção de lombalgia e do exercício físico em trabalhadores de escritórios.

Métodos: A investigação parte de uma análise de artigos já publicados, embasada em uma busca de estudos na base de dados BVS, recorrendo aos descritores: ginástica laboral e lombalgia. Selecionou-se 8 artigos, os quais compreendem a amostra final desta revisão.

Resultados: As evidências registradas nos diferentes artigos encontrados evidenciam a importância do programa de Ginástica Laboral na prevenção de dor nas costas ou lombalgia no ambiente de trabalho.

Conclusão: A Ginástica Laboral contribui efetivamente para que pessoas tenham uma melhoria de vida.

Larisse Freitas Soares da Costa

Graduanda em Educação Física pela AESPI - Ensino Superior do Piauí

Teresina - Piauí

iD https://orcid.org/00000002-6931-8821

Thanandra Rocha Ferreira

Profissional de Educação Física, Mestre e Professor da Faculdade AESPI - Ensino Superior do

Piauí

Teresina - Piauí

in https://orcid.org/00000001-7005-9102 


\section{LABORAL GYMNASTICS IN THE}

PREVENTION OF (OCCUPATIONAL)

LOM-BALGY IN THE WORKPLACE:

integrative review

DOI: $10.48140 /$ digitaleditora.2020.002.3

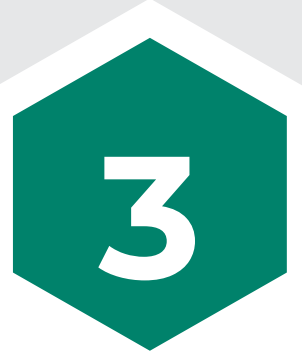

\section{ABSTRACT}

Recebido em: 10/12/2020

Aprovado em: 28/12/2020

Conflito de Interesse: não

Suporte Financeiro: não houve
Objectives: The objective of this study is to analyze, through a bibliographic review, the importance of the labor gymnastics program in the prevention of low back pain and physical exercise in office workers.

Methods: The investigation starts from an analysis of articles already published, based on a search for studies in the VHL database, using the descriptors: gymnastics and low back pain. Eight articles were selected, which comprise the final sample of this review Results: The evidence recorded in the different articles found shows the importance of the Labor Gymnastics program in the prevention of back pain or low back pain in the workplace.

Conclusion: Gymnastics at work contributes effectively for people to improve their lives. 


\section{INTRODUÇÃO}

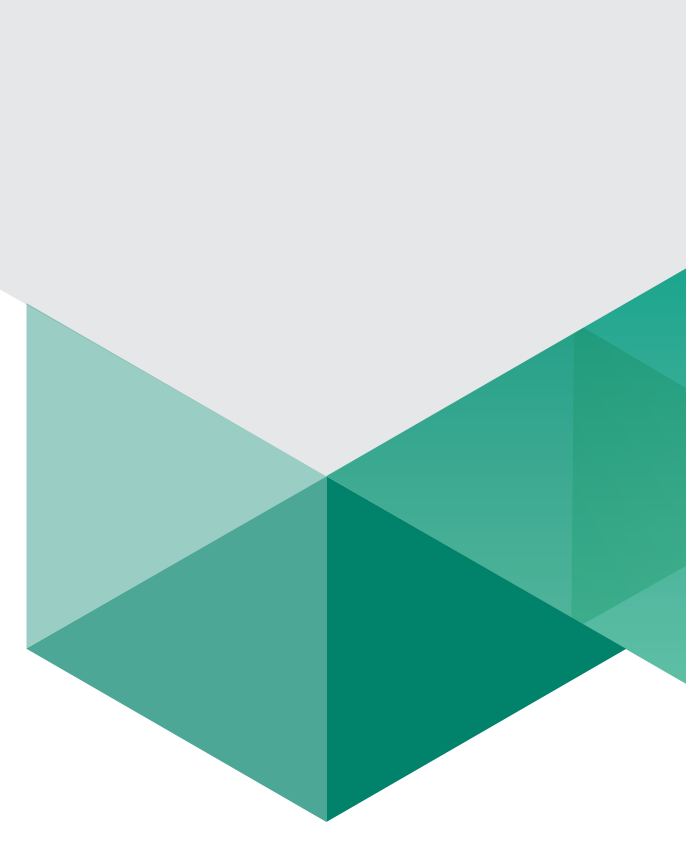

A vida humana, ao longo dos anos, vem tentando acompanhar o ritmo frenético em que o mundo se globaliza e disponibiliza novos avanços tecnológicos. Desta maneira, o homem moderno ressignifica suas necessidades, expandindo valores e aderindo a novos estilos de vida. À medida em que as pessoas começam a consumir mais e almejar outros padrões sociais; tendem a aumentar o período em que passam exercendo uma atividade laboral, consequentemente expandem o tempo de carga horária de trabalho, tendo dupla jornada, outro emprego no contra turno ou até mesmo fazendo hora extra; visando ter um melhor rendimento no capital, esquecendo de cuidar do bem-estar (ÁVILA E SANTOS,1999).

Com essas mudanças, a vida cotidiana passa a ter alterações na área da saúde e no desenvolvimento do trabalho. Na saúde, por desencadear lesões através de movimentos repetitivos; no trabalho, refletindo na baixa produtividade, afastamento por atestados médicos; problemas que sobrecarregam o organismo físico e mental. Os movimentos repetitivos, no qual o trabalhador se utiliza, compreendendo sempre os mesmos segmentos corporais, contribuem para o aumento das doenças do trabaIho, específicas em cada categoria profissional, agravadas pelo sedentarismo, estresse e sobrecarga de atividades. (FERNANDES et al., 2011)

Segundo Briganó e Macedo (2005), o termo lombalgia designa a dor na coluna lombar, esta dor abrange tanto o sexo masculino, quanto o feminino, variando entre uma dor súbita e uma dor intensa e distendida. Ocorre frequentemente nas atividades laborais, não se referindo a doença, mas sim, um indício que pode levar a alguma patologia. Contudo, o mundo do trabalho tem sofrido mudanças tecnológicas e organizacionais, exigindo constantemente que os trabalhadores se adaptem às tecnologias e se atualizem perante um mercado cada vez mais competitivo (REIS et al., 2011), trazendo tanto para os funcionários como para as empresas benefícios, vantagens que incluem uma boa qualidade de vida e grande produtividade para um significativo rendimento em capital. Vários estudos têm demonstrado que o estresse imposto pelas longas jornadas de trabalho, geralmente decorre de más posturas, utilização de mobílias inadequadas, prevalência de lombalgia e problemas posturais (PASTRE et al., 2011).

Apenas algumas empresas e instituições estão preocupadas em oferecer aos seus colaboradores condições ideais de trabalho, não estando a grande maioria preocupada em investir na melhoria 
da qualidade de vida de seus funcionários, mas somente com a produtividade dos mesmos. É fundamental identificar os fatores de risco de lombalgia, que incluem características ocupacionais ou psicológicas. Ocupacionais, quando o funcionário acarreta para si uma quantidade de carga (peso) acima do possível para seu corpo, ocasionando também um dano psicológico que acaba direcionando para quadros depressivos, tendo o mesmo que se afastar por períodos prolongados da empresa para tratar-se (FUNDACENTRO, 2007).

Com o fim de analisar esse campo, este estudo trata da lombalgia e sua prevenção através da ginástica laboral, o quanto é eficiente na melhoria das dores e da qualidade de vida. A ginástica laboral surge como uma tentativa de contornar esses problemas. As empresas vêm adotando a ginástica laboral (GL), como sendo uma possibilidade de diminuir afastamentos decorrentes de lesões (NAHAS, et al., 1992) para uma redução de atestados médicos e aumento da produtividade. A inserção da $G L$ na rotina das empresas pode apresentar resultados positivos, como o aumento da produtividade e disposição para o trabalho, melhoria da qualidade de vida e conscientização corporal, Pressi e Candotti (2011) reforçaram essa ideia. A GL com-preende exercícios específicos de alongamento, de fortalecimento muscular, de coordenação motora e de relaxamento, realizados em diferentes setores ou departamentos da empresa, tendo como objetivo principal prevenir e diminuir os casos de LER/DORT (OLIVEIRA, 2007).

Destarte, o programa de GL apresenta-se como recurso terapêutico para a lombalgia por ter o intuito de refrear lesões que surgem a partir do exercício do tra-balho. Assim, este programa busca proporcionar benefícios tanto para o trabalhador quanto para a empresa, considerando-se que mesmo com uma pequena pausa du-rante o período de trabalho para atividade da GL, é possível perceber benefícios que repercutem na produtividade da empresa e consequentemente conseguem prevenir as dores ocasionadas por conta do exercício do trabalho. A lombalgia é definida como intolerância à atividade devido os sistemas lombares estarem associados a sintomatologia nos membros inferiores. Pode ser classificada em aguda e crônica em virtude principalmente do fator tempo. Refere-se a dor causada em indivíduos que passam um longo período sentados, estando assim, ligada também ao sedentarismo (TOSCANO \& EGYPTO, 2001). A dor aguda é percebida quando no indivíduo manifesta-se apresentando duração de quatro a seis semanas, e não está relacionada a nenhum fator definido, geralmente indicando melhoras da dor em 4 semanas. Já a crônica, ocorre em qualquer idade e em aproximadamente $75 \%$ a $85 \%$ das pessoas que se afastam do trabalho com dores mais intensas e sem condições de voltar ao trabalho.

Apercebe-se que a realidade de cada função empreendida ao decorrer do itinerário de trabalho pode gerar tanto benefícios, quanto provocar o aparecimento de doenças ocupacionais, tais como lesão por esforço repetitivo, que foi denominada como doença osteomuslar relacionada ao trabaIho, fadiga nos membros, dentre elas destaca a lombalgia (ALMEIDA et Al., 2008 apud NASCIMENTO, 2015). A lombalgia é uma doença que se manifesta em inúmeras pessoas, principalmente em funcionários quem tem uma demanda de carga horária com movimentos repetitivos, ficando sentados por um longo período.

A lombalgia pode ocasionar danos terríveis às pessoas, a exemplo: afetar o lombar de uma maneira que o indivíduo não possa mais caminhar. O programa de ginástica laboral quando executado de maneira preventiva pode favorecer a redução do quadro de lombalgia ocupacional no ambiente de trabalho. Dessa forma, a pesquisa tenta responder à questão norteadora: A existência de um exercício físico por um determinado tempo pode prevenir o aparecimento da lombalgia e quais efeitos positivos propicia na recuperação do paciente com essa síndrome?

O Programa da ginástica laboral tem efeitos satisfatórios em pessoas que apresentam lombal- 
gia, pois previne e ameniza as dores frequentes com intensidade e também auxilia na correção de postura do indivíduo enquanto sentado. Em virtude disso, o objetivo geral desse estudo é analisar os efeitos da ginástica laboral sobre as dores lombar ou lombalgia ocasionadas no ambiente de trabalho desencadeadas por movimentos repetitivos de quem fica por um longo período na posição sentada. Delimitou-se como objetivos específicos: avaliar a ação da ginástica laboral em pes-soas que tem lombalgia por trabalharem em tempo excessivo, sentadas na mesma posição por um determinado período. Registrar os efeitos positivos da ginástica labo-ral, durante o tempo de exercícios executados no ambiente de trabalho. Comparar os efeitos da ginástica laboral em funcionários que tem uma rotina de trabalho na postura sentada, colocado por diferentes autores. Por último, identificar a aplicabili-dade do programa de ginástica laboral, dentro das empresas, em funcionários que atuam na área administrativa (escritório) diante de suas demandas para sua eficácia.

As empresas se alicerçam por meio do trabalho de pessoas, bem como elas se adaptam às novas tecnologias e exigências do mercado. Por isso, esse trabalho justifica-se ao tratar dos riscos à saúde diante da exposição de um setor corporativo a rotinas de trabalho que condicionam e acomodam pessoas, tornando-as alheias aos cuidados adequados com seu bem-estar e a importância da conscientização e implantação de programas que atendam às necessidades funcionais de seus colaboradores. Observando-se a importância da atividade física mediada por um profissional da área que traga um instrumental de treinos diários que atendam as particularidades de cada profissional de forma geral.

\section{METODOLOGIA| TIPO DE ESTUDO}

A metodologia se restringe a uma análise descritiva simples compreendendo um conjunto de reflexões teóricas sobre um tema. O presente estudo constitui-se como uma pesquisa de revisão integrativa de literatura. A revisão integrativa configura-se como um tipo de análise que reúne achados de estudos desenvolvidos mediante diferentes metodologias, permitindo aos revisores sintetizar resultados sem ferir a filiação epistemológica dos estudos empíricos incluídos. Para a elaboração da presente revisão, serão utilizados apenas estudos experimentais (humanos), tanto no delineamento randomizado, quanto não randomizado e aqueles que estejam indexados em revistas da plataforma PEDro. A revisão integrativa é o método mais genérico, permitindo a inserção paralela da pesquisa empírica e a que se aproxima da empírica, proporcionando um entendimento maior do tema de relevância. Este processo permite o arranjo de informações tanto da literatura teórica, quanto do conhecimento empírico.

\section{COLETA DE DADOS}

Realizou-se uma revisão integrativa das produções científicas em torno do tema "Iombalgia e ginástica laboral" publicados entre os anos de 2007 a 2020, últimos 13 anos. A seleção dos artigos foi realizada no mês junho de 2020, nas bases de dados da Biblioteca Virtual em Saúde 
(BVS): MedLine (Medical Literature Analysis and Retrieval System Online), LiLacs (Latin American and Caribbean Health Sciences Literature) e SciELo (Scientific Electronic Library Online). As bases de dados referenciadas foram escolhidas por apresentarem fiabilidade aos temas pesquisados, sendo espaços multíplices de divulgação científica e base fundamental e precisa para a produção técnica.

Conforme o levantamento feito nas bases de dados recuperou-se um total de 20 artigos pesquisados e apenas 8 foram selecionados para análise. As buscas fo-ram realizadas sem restrição de idiomas e deram-se de forma que se excluiu os arti-gos de revisão sistemática, literatura, bibliográfica, teses e dissertações, publicações fora do período de busca pré-estabelecida, evidências com possíveis resultados ori-undos de viés. A autora selecionou os estudos para inclusão, baseando-se na leitura e análise crítica dos títulos e resumos. Os artigos foram classificados em pesquisas adequadas (quando os estudos estavam adequados aos critérios de elegibilidade) e inadequadas (os que não se enquadraram aos objetivos propostos). A pesquisadora extraiu os dados, avaliou a qualidade metodológica e analisou os resultados de forma cegada.

\section{CRITÉRIOS DE INCLUSÃO E EX- CLUSÃO DOS ESTUDOS}

Os termos utilizados foram adequados de acordo com os descritores de ciên-cias e saúde (DesCs): (ginastica laboral, lombalgia, ginastica laboral ocupacional, prevenção). Os quais foram combinados com descritores booleanos (or, and e not). A pesquisa orientou-se respeitando os critérios de inclusão definidos para a seleção dos artigos: (1) Estar relacionado à temática "lombalgia e ginástica laboral", (2) Ter sido publicado entre 2007 a 2020, (3) Trazer um delineamento dos resultados da prática da ginástica laboral. Os artigos excluídos, no que Ihes concerne, abrangiam temas que não se relacionavam com a proposta de investigação do trabalho, apresentando: (1) Conteúdo que não apresentavam correlação com a minimização de dores lombar, (2) Não apresentarem resultados para o trabalho com a ginástica laboral.

\section{ANÁLISE DOS DADOS}

Os dados dos estudos foram analisados e catalogados em uma tabela pré-estabelecida, os quais compreendem os seguintes itens: autor/ano, revista publica-da, objetivo, amostra, metodologia, tempo experimental e resultados principais. 
Figura 1 . Fluxograma de procedimento metodológico para seleção de artigos.

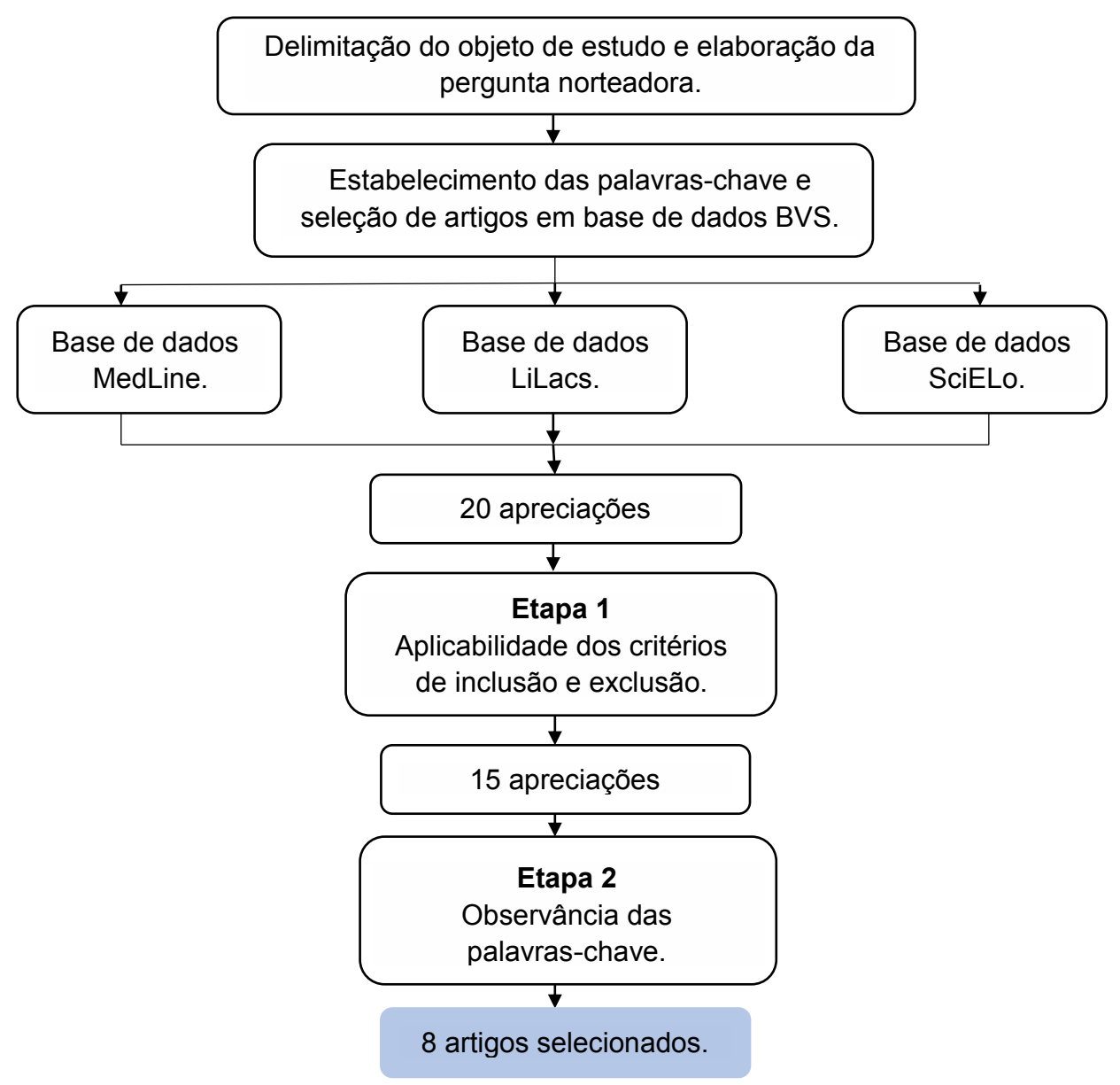

\section{RESULTADOS}

A metodologia de pesquisa qualitativa tem caráter exploratório dentro do estudo dos fenômenos que envolvem o humano e a complexidade de suas interações sociais. Este estudo parte de uma perspectiva de análise integrada, considerando a dinâmica do evento observado. Constitui uma pesquisa documental, desenvolvida por meio do exame de conteúdo. Faz-se adequada enquanto método investigativo, pois permite o acesso a dados de pessoas as quais não se pode ter acesso material, devido a fatores que impossibilitem o contato direto. A investigação através de documentos é acertada no momento em que objetivamos analisar um amplo interva-lo de tempo, na busca de verificar um ou mais eventos no comportamento de um determinado fenômeno (GODOY, 1995, p. 20-29).

Candotti (2010), afirma que o estresse por conta de intensas jornadas de trabalho, seguido de má postura ocasiona a presença de lombalgia. A ginástica laboral existe desde o ano de 1925 na Polônia, conhecida como ginástica de pausa. Na contemporaneidade a ginástica laboral está sendo muito utilizada nas empresas como meio de amenizar dores causadas por movimentos repetitivos. Nesse estudo foram utilizados artigos onde diferentes autores apresentam a lombalgia como uma patologia comum em trabalhadores que passam muito tempo em uma só posição, afetando assim o membro inferior.

A pesquisa de dados feita nas plataformas de publicação científica fundamentou a análise permitindo averiguar a hipótese levantada e retorquir os objetivos delineados. O corpus de estudos analisados consta em número reduzido, devido a quantidade de achados, levando em questão os critérios de 
seleção e a pergunta norteadora mediante a avaliação da ginástica laboral como mecanismo interventor no quadro de pessoas que apresentam lombalgia, bem como o destaque de seus efeitos positivos, comparando os resultados a partir da abordagem de diferentes autores mediante sua implantação e efitividade no local de trabalho.

Nesta investigação, foram encontrados quatro artigos que relatam a melhoria no bem-estar de funcionários da área administrativa que praticam a ginástica laboral por no máximo 15 minutos ao dia, variando a quantidade de vezes por semana e meses por ano, dentro das empresas em que trabalham, com impacto direto na região lombar. Dois estudos analisados nessa revisão demarcaram a inclusão não apenas de funcionários do corpus administrativo, mas também dos funcionários de forma geral para a prática da ginástica laboral. Um dos estudos selecionados desta-cou a importância da ginástica laboral mediante a prevenção de doenças ocupacionais.

O Quadro 1 apresenta uma síntese dos estudos encontrados de forma que se notabiliza: autor e ano, revista publicada na qual o artigo foi publicado, objetivo geral, amostra, metodologia, tempo experimental e resultados principais.

QUADRO 1. Resultado dos estudos de avaliação dos efeitos e benefícios da Ginástica Laboral em trabalhadores.

\begin{tabular}{|c|c|c|c|c|c|}
\hline AUTOR/ ANO & REVISTA & OBJETIVO & AMOSTRA & INTERVENÇÃO & RESULTADO \\
\hline FERREIRA/2013 & $\begin{array}{l}\text { E d u c a ç ã o } \\
\text { Física UNI- } \\
\text { FAFIBE }\end{array}$ & $\begin{array}{l}\text { Verificar os benefí- } \\
\text { cios da GL e iden- } \\
\text { tificar os possíveis } \\
\text { motivos de sua não } \\
\text { implantação. }\end{array}$ & Trabalhadores. & $\begin{array}{l}\text { Treinando todos os dias } \\
\text { com duração de } 5 \text { a } 7 \\
\text { minutos com treinos } \\
\text { apropri-ado. }\end{array}$ & $\begin{array}{l}\text { A implantação da } \\
\text { GL beneficia a em- } \\
\text {-presa na redução } \\
\text { dos afastamento } \\
\text { do trabalho. }\end{array}$ \\
\hline CRUZ/2014 & $\begin{array}{l}\text { Latino Ame- } \\
\text { ricana de En- } \\
\text { fermagem }\end{array}$ & $\begin{array}{l}\text { Avaliar o efeito de } \\
\text { um progra-ma de } \\
\text { GL com-pensató- } \\
\text { rio, visando a re- } \\
\text {-dução do es-tres- } \\
\text { se ocupa-cional. }\end{array}$ & $\begin{array}{l}\text { Trabalhadores } \\
\text { Administrati- } \\
\text { vos. }\end{array}$ & $\begin{array}{l}\text { Treinos com fre- } \\
\text { quência de } 2 \text { vezes } \\
\text { por semana, com } \\
\text { duração de } 15 \text { mi- } \\
\text { nutos cada, por } 10 \\
\text { sema-nas. }\end{array}$ & $\begin{array}{l}\text { O programa de } \\
\text { GL promoveu a } \\
\text { redução de lom- } \\
\text { balgias osteo- } \\
\text { musculares na } \\
\text { maioria dos seg- } \\
\text { mentos cor-po- } \\
\text { rais avaliados. }\end{array}$ \\
\hline CUNHA/ 2016 & $\begin{array}{l}\text { Ciência\&Tra- } \\
\text { balho }\end{array}$ & $\begin{array}{l}\text { Analisar a influên- } \\
\text { cia de um progra- } \\
\text { ma de GL na re-du- } \\
\text { ção de ates-tados } \\
\text { de uma empresa. }\end{array}$ & $\begin{array}{l}\text { Trabalhadores } \\
\text { administrativos } \\
\text { e de produção. }\end{array}$ & $\begin{array}{l}\text { Foi desenvolvi-do } 2 \\
\text { vezes na se-mana } \\
\text { com duração de } 10 \\
\text { minutos por } 12 \text { me- } \\
\text { ses con-tínuos. }\end{array}$ & $\begin{array}{l}\text { Teve uma dimi- } \\
\text {-nuição do ab- } \\
\text { senteísmo em } \\
\text { ambos os sexos } \\
\text { durante o pro- } \\
\text { grama de GL. }\end{array}$ \\
\hline OLIVEIRA/ 2007 & $\begin{array}{l}\text { Educação Fí- } \\
\text { sica }\end{array}$ & $\begin{array}{l}\text { A importância da } \\
\text { GL na pre-venção } \\
\text { de doenças ocu- } \\
\text {-pacionais. }\end{array}$ & $\begin{array}{l}\text { Trabalhadores } \\
\text { Administrati- } \\
\text { vos. }\end{array}$ & $\begin{array}{l}\text { Foi efetuado sessões } \\
\text { de } 5,10 \text { e } 15 \text { minu- } \\
\text { tos durante } 12 \text { me- } \\
\text { ses. }\end{array}$ & $\begin{array}{l}\text { Evidências de- } \\
\text {-monstram a im- } \\
\text { portância da GL } \\
\text { na prevenção de } \\
\text { doenças ocupa- } \\
\text {-cionais. }\end{array}$ \\
\hline CANDOTTI/2010 & $\begin{array}{l}\text { Brasileira de } \\
\text { Ciências do } \\
\text { Es-porte }\end{array}$ & $\begin{array}{l}\text { Verificar o efeito da } \\
\text { GL sobre dor nas } \\
\text { costas e os hábitos } \\
\text { posturais. }\end{array}$ & $\begin{array}{l}\text { Trabalhadores } \\
\text { Administrati- } \\
\text { vos. }\end{array}$ & $\begin{array}{l}\text { Cada sessão com du- } \\
\text { ração de } 15 \text { minutos, } \\
3 \text { vezes na se-mana } \\
\text { por } 3 \text { meses. }\end{array}$ & $\begin{array}{l}\text { Foi eficaz na di- } \\
\text { minuição da in- } \\
\text { tensidade da dor } \\
\text { e na corre-ção } \\
\text { dos hábitos pos- } \\
\text { turais. }\end{array}$ \\
\hline
\end{tabular}

Fonte: Pesquisa realizada em bases de dados. 


\section{DISCUSSÃO}

Os estudos que compreendem essa análise revelam os benefícios propiciados pela ginástica laboral em detrimento aos riscos ergonômicos desencadeados pela atividade de trabalho. A implantação da ginástica laboral atende as prescrições da NR-17 que visa o estabelecimento de parâmetros para uma adaptação do ambiente de trabalho às características psíquico e fisiológicas dos funcionários de empresas, onde cabe ao empregador realizar a análise ergonômica do ambiente onde a atividade laboral é desenvolvida.

Candotti, verificou o efeito da ginástica laboral sobre a dor nas costas e maus hábitos posturais em trabalhadores que ficam longos períodos na posição sentada, contando com a participação de 30 trabalhadores de ambos os gêneros de uma em-presa em Portão-RS. O PGL (Programa de Ginástica Laboral) foi realizado durante três meses consecutivos, com sessões de 15 minutos, três vezes por semana; com ênfase nos membros superiores e coluna cervical. Os resultados demonstrados através de dados coletados do grupo experimental e do controle asseveraram que a implementação do PGL foi eficaz, diminuindo a intensidade e frequência das dores, e na correção dos hábitos posturais, proporcionando aos participantes ter uma melhor postura sentada.

Oliveira, realizou um estudo no qual fez um levantamento sobre o histórico das Lesões por Esforços Repetitivos (LER) e os Distúrbios Osteomusculares Relacionados ao Trabalho (DORT), e a importância do PGL na prevenção de doenças ocupacionais. Observou os efeitos da implementação da atividade de ginástica laboral em empresas que adotaram a prática de forma integral para seus colaboradores em um intervalo de tempo de três anos consecutivos. Destacou como benefícios da incorporação do PGL, uma redução dos casos de LER/DORT, baixo dispêndio com auxílio médico, atenuação das dores corporais, redução das faltas no local de trabalho, novas perspectivas de estilo de vida e o aumento do bom desempenho na ativi-dade de trabalho. Embora, a ênfase nos aspectos benéficos do investimento das empresas na aplicação do PGL, o estudo demonstrou que no Brasil os dados sobre o retorno financeiro para algumas empresas que implementam o PGL, não são divulgados por essas instituições, pois embora traga um retorno positivo, essas enti-dades não querem a implementação obrigatória do programa.

Segundo Moreira, que efetuou um estudo randomizado em 15 colaboradores da empresa CARAMURU ALIMENTOS S/A, situada no município de Apucarana/PR, que apresentavam queixa de lombalgia, através da execução do PGL por quatro semanas seguidas, três vezes por semana, verificou que as sessões favoreceram em um grupo que se distribuía em cinco mulheres e dez homens, com idades entre 22 e 57 anos, os efeitos da ginástica nas mulheres foi na variação e alteração na intensidade da dor para dor ligeira a sem dor, os homens, afirmaram o mesmo, e ainda relataram que houve melhoria efetiva do bem-estar e humor ao decorrer da jornada de trabalho.

Ferreira aponta que ginástica laboral (GL) viabiliza a melhora da qualidade de vida dos funcionários e o rendimento produtivo da empresa. Buscou averiguar os benefícios da GL tanto para o funcionário, quanto para a empresa, e identificar as possíveis causas de sua não implantação. Boa parte dos resultados foram satisfatórios após o exercício da $\mathrm{GL}$, dentre eles constata-se a melhora na qualidade de vida e na autoestima dos funcionários, a redução das faltas e dos afastamentos médicos por lesões causadas no trabalho, além da redução do percentual de adipose e das contingencias que podem ocorrer na hora do expediente.

O estudo de Laux, mostrou que em uma amostra composta por 31 sujeitos, sendo 22 do sexo masculino e 9 do sexo feminino com idade média de 28 a 48 anos, funcionário de uma empresa de 


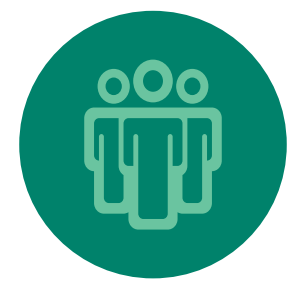

\section{CONCLUSÃO}

A atividade física realizada no local de trabalho feita através de um programa de recuperação, manutenção da qualidade de vida e fomento ao lazer desempenhada de maneira assertiva, quando pensada de acordo com público alvo, dá-se como prática de prevenção e reabilitadora. Com base em um plano simples de atividades diárias orientados por um profissional adequado, apercebe-se que o grupo corporativo das empresas analisadas, apresentam uma melhoria significativa tanto no tratamento das dores lombares, quanto na prevenção de um quadro clínico de lombalgia, visto que as atividades realizadas proporcionam uma maior consciência corporal e mobilidade.

Os benefícios observados com inserção do PGL (programa de ginástica laboral) implicam no combate a doenças como LER, DOR, lombalgia, dentre outras que afetam o colaborador, que está alheio aos riscos que advém da lida com o trabalho, proporcionando a prevenção de problemas osteomusculares, o fortalecimento mus-cular; instigando ao trabalhador na adoção de posturas adequadas e a prática de atividade física, dinâmica; seja ela individual ou coletiva. Objetivando assim, otimizar rotinas de trabalho, minimizar a sensação de fadiga ao final do expediente, coibir eventuais acidentes, elevar a produtividade.

Para tanto, faz-se necessário que as empresas implementem como parte de um projeto de saúde no trabalho, um programa que abranja desde a conscientização de seus colaboradores em relação a bons hábitos posturais ao exercerem suas funções, ao exercício efetivo da prática coordenada de atividades que auxiliem na boa manutenção do corpo, como também no desempenho de suas atividades. O PGL tem se mostrado eficaz nas empresas que resolveram efetivar essa técnica. Cabe as empresas, ademais, equipar suas dependências com mobília adequada e confortável, pensando na permanência por hora de cada funcionário em determinada posição.

Oferecer condições salubres de trabalho e cuidar de seus cooperados é socializar não só referências de trabalho humanizado como também assegurar a confiabilidade tanto de seus prestadores, como de seu público-alvo, passando uma responsabilidade social valorativa. De acordo com esse estudo, observou-se que, o programa de Ginástica Laboral, tem efeitos satisfatórios, podendo desse modo, re-duzir e prevenir patologias decorrentes da postura em que se desenvolve as atividades no ambiente de trabalho. A Ginástica Laboral contribui efetivamente para que pessoas tenham uma meIhoria de vida. 


\section{REFERÊNCIAS}

ÁVILA, C. A. V.; SANTOS, J. B. dos. Estudo da distribuição da pressão exercida sobre a região glútea em costureiras da Indústria Têxtil Sulfabril Blumenal/SC. In: CONGRESSO BRASILEIRO DE ERGONOMIA, 9, 1999, Salvador. Anais... Rio de Janeiro, 1999.

CANDOTTI, Cláudia Tarragô; STROSCHEIN, Rosemeri; NOLL, Matias. Efeito da ginástica laboral na dor nas costas e nos hábitos posturais adotados no ambiente de trabalho. Revista Brasileira de Ciências do Esporte, v. 33, n.2, 2011.

COURY, Helenice; MOREIRA, Roberta; DIAS, Natália. Efetividade do exercício físico em ambiente ocupacional para controle da dor cervical, lombar e do ombro: uma revisão sistemática. Revista brasileira de fisioterapia, v.3, 2019.

DE OLIVEIRA, João Ricardo Gabriel. A IMPORTÂNCIA DA GINÁSTICA LABORAL NA PREVENÇÃO DE DOENÇAS OCUPACIONAIS. Revista de Educação Física, 2007; 139:40-49.

DOS SANTOS, Andréia Fuentes; ODA, Juliano Yasuó; NUNES, Ana Paula Mori; GONÇALVES, Luciano; GARNÉS, Fabrícia Lorca dos Santos. BENEFíCIOS DA GI-NÁSTICA LABORAL NA PREVENÇÃO DOS DISTÚRBIOS OSTEOMUSCULARES RELACIONADOS AO TRABALHO. Arq. Ciênc. Saúde Unipar, v.11, n.2, 2007.

FERREIRA, Karina da Silvia; DOS SANTOS, André Pereira. OS BENEFÍCIOS DA GINÁSTICA LABORAL E OS POSSÍVEIS MOTIVOS DA NÃO IMPLANTAÇÃO. Re-vista Educação física UNIFAFIBE, n.2, p.56-72, 2013.

FUJIMOTO, Heyla Medeiros Gualberto; AVELAR, Solange Andrade. INFLUÊNCIA DA GINÁSTICA LABORAL NA MINIMIZAÇÃO DO ESTRESSE OCUPACIONAL. Revista Enfermagem Integrada, v.5, n.2, 2012.

FUNDACENTRO. Ministério do Trabalho e Emprego. LER/ DORT.

GODOY, A. S. Pesquisa qualitativa: tipos fundamentais. Revista de Administração de Empresas, v. 35, n. 3, p. 20-29, 1995.

LAUX, Rafael Cunha; PAGLIARI, Paulo; JUNIOR, João Viannei Effting; CORAZZA, Sara Teresinha. Programa de Ginástica Laboral e a Redução de Atestados Médicos. Revista Ciência e Trabalho, 2016.

MOREIRA, Bruna de Almeida; ANDOLFATO, K. R. Os efeitos da ginástica laboral sobre as lombalgias ocupacionais. In: CONGRESSO MULTIDISCIPLINAR. Anais eletrônicos. Apucarana: 2018.

NAHAS, M. V.; CORBIN, C. B. Educação para aptidão física e saúde: justificativa e sugestões para implementação nos programas de educação física. Revista brasilei-ra de Ciência e Movimento. 8 (3): 14-24, 1992.

SERRA, Maysa Venturoso Gongora Buckeridge; PIMENTA, Lorrana Campos; QUEMELO, Paulo Roberto Veiga. EFEITOS DA GINÁSTICA LABORAL NA SAÚDE DO TRABALHADOR. Revista Pesquisa de fisioterapia, 2014.

SILVA, Sarah Etienne Arreguy Rodrigues . Ginástica laboral: uma perspectiva de promoção de saúde. Universidade Federal de Minas Gerais. Faculdade de Medicina. Núcleo de Educação em Saúde Coletiva . Governador Valadares, 2014. 